\title{
DIGITAL DIVIDE IN INTERNET ACCESS AMONG LITERATE STUDENTS UNTIL ENTERING UNIVERSITY: A BRAZILIAN PROJECT PROPOSAL
}

\author{
Juliao Braga \\ FCI \\ Universidade Presbiteriana Mackenzie \\ Sao Paulo, SP Brazil \\ j@braga.net.br \\ Ana Leda Silva Moraes \\ PPG-CC \\ State Federal University of Sao Paulo \\ Sao Jose dos Campos, SP Brazil \\ analedamoraes@gmail.com
}

\author{
Jeferson Nobre \\ Institute of Informatics \\ Federal University of Rio Grande do Sul \\ Porto Alegre, RS Brazil \\ jcnobre@inf.ufrgs.br \\ Marcelo Santos \\ Computer Science Department \\ Federal Institute of Sertao Pernambucano \\ Salgueiro, PE Brazil \\ marcelo.santos@ifsertao-pe.edu.br
}

October 11, 2020

\begin{abstract}
This work describes a project that aims to reduce the opportunities divide in Internet access in Brazil, but that can be used to meet a similar objective in other countries. Basic education students, living in peripheral communities or in remote locations and participating in rural public schools, among others, have immense difficulties in accessing the Internet. As a result, they no longer participate in the largest repository of knowledge in the world and lose favorable occasions for their personal growth and consequent encouragement to continue to overcome obstacles and continue in the serene construction of a future of their choice.
\end{abstract}

\section{Introduction}

This paper describes a project that aims to reduce the opportunities divide in Internet access in Brazil, but that can be used to meet a similar objective in other countries.

Elementary and high school students, living in peripheral communities or in remote locations, and participating in rural public schools, among others, have immense difficulties in accessing the Internet. As a result, they no longer participate in the largest repository of knowledge in the world and lose favorable occasions for their personal growth and consequent encouragement to continue to overcome obstacles and continue in the serene construction of a future of their choice.

This project, called ITER Project (ITER), aims to address this inequality, considering:

- Low implementation and operation cost

- Capillarity

- speed of implementation

- Security

producing actions that make effective:

- the availability of Internet access for students, from literate students until entering University; 
- to provide and maintain Internet traffic in public secondary schools;

- provide and keep Internet traffic in public secondary schools, in community settings, and at student homes;

- support for community Internet networks, without direct involvement with the respective operational activities.

ITER proposes to meet the objectives outlined above, with two parallel actions:

1. Facilitate the use of the Internet through the more than 2,600 eduroam locations in Brazil;

2. Use of the immense access capillarity of small, medium, and large Internet Service Providers (ISPs), duly remunerated for this initiative.

To ensure the viability of these objectives, ITER will undergo a test implementation during the period of fifteen (15) months, throughout Brazil. This implementation will be called Proof of Concept (PC). This will happen through a joint effort by Employees and Partners (individuals and companies) recording the lessons learned during PC and displaying their results in the smallest details. From these experiences and notes, ITER will be improved. The ITER information repository is available in the Open Science Framework ${ }^{1}$ (OSF).

This paper is a compilation and translation of excerpts from the article [1]. It is based on studies done in Brazil, where there are about 38,450,909 students who should have access to the Internet. Estimates indicate that around $60 \%$ do not have this access or have very restricted access. The map in Figure 1 displays this number divided by Brazilian state.

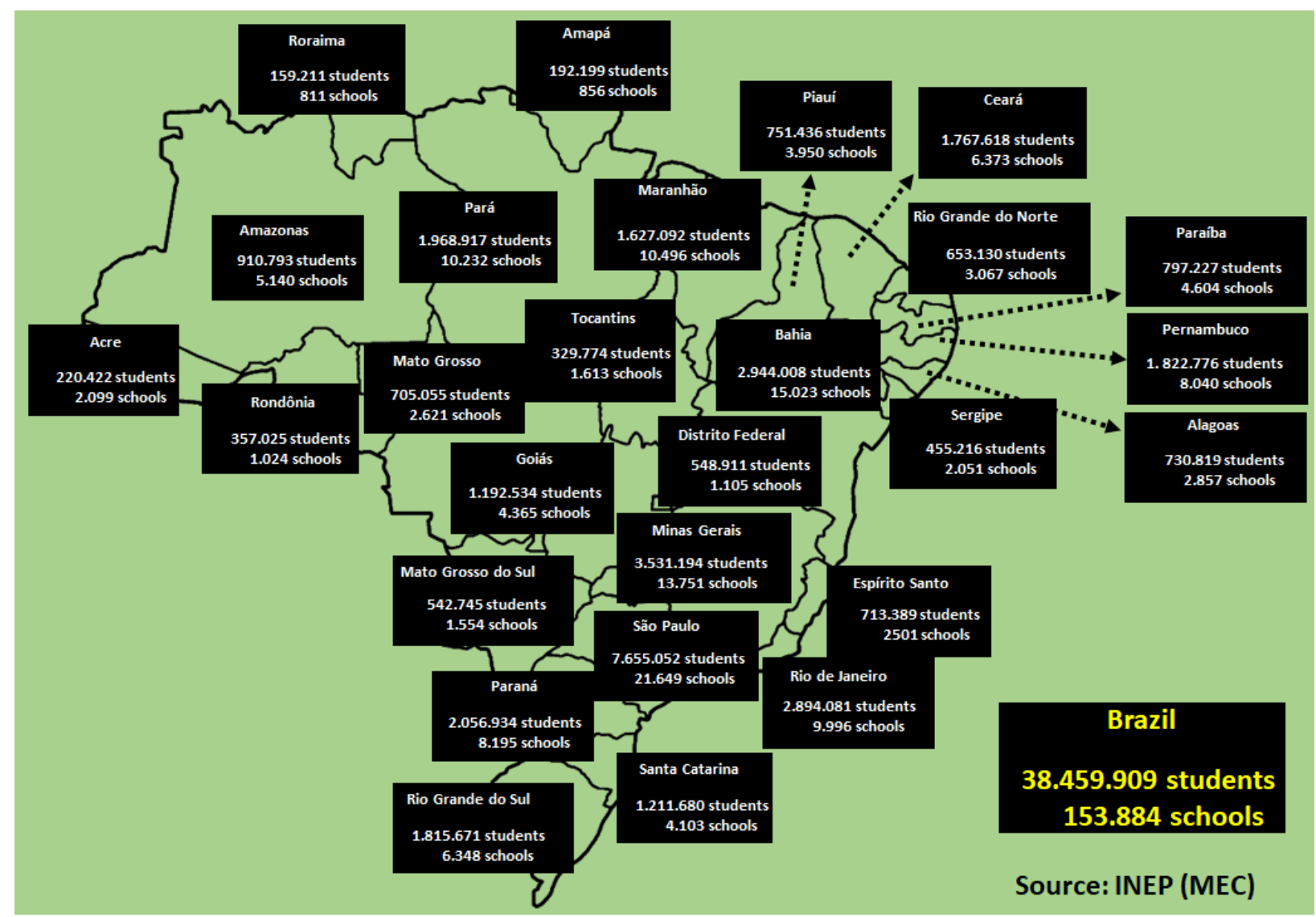

Figure 1: Brazilian students and schools, by state

This work is divided into seven sections, including this Introduction. Section 2 is a summary presentation of eduroam. Section 3 presents the topology of ITER and its relationship with eduroam, and Internet Access Providers (ISPs) addressing the RADIUS proposal as a solution to the ITER architecture proposal described in Section 4. Section 5 presents ITER's operational strategies, its governance and the participation of multiple stakeholders, characterizing the

${ }^{1}$ https://osf.io/mrkvw/ 
institutional support of ISOC Brazil (Brazilian chapter of the Internet Society). Section 6 presents the initial steps to implement the project and to establish the first governance documents. Finally, Section 7 presents a summary of the conclusions and future work associated with the project.

\section{O eduroam}

eduroam is an initiative created in 2003 and is now operating in more than 100 countries, with a strong academic orientation [2]. Its presence can be seen in Figure 2.

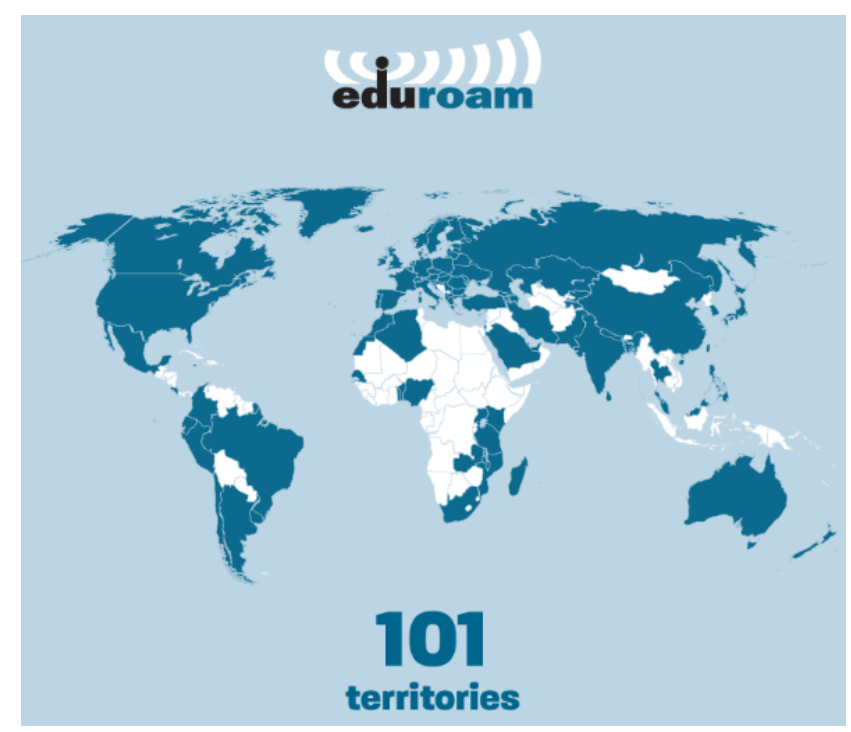

Figure 2: eduroam's presence in the world. Source: https://www.eduroam.org/wp-content/uploads/ eduroam-growing-topology-v2.gif

It is a technology based on the IEEE 802.1X standard and on a set of servers based on the RADIUS protocol (RADIUS is an acronym for Remote Access Dial In User Service), defined by the Internet Engineering Task Force (IETF), in 1997 described and updated by RFC2865. [3].

Techniques available in RADIUS enable facilities that eduroam explores and that we can see in its operation, as shown in Figure 3 and by the explanations that follow.

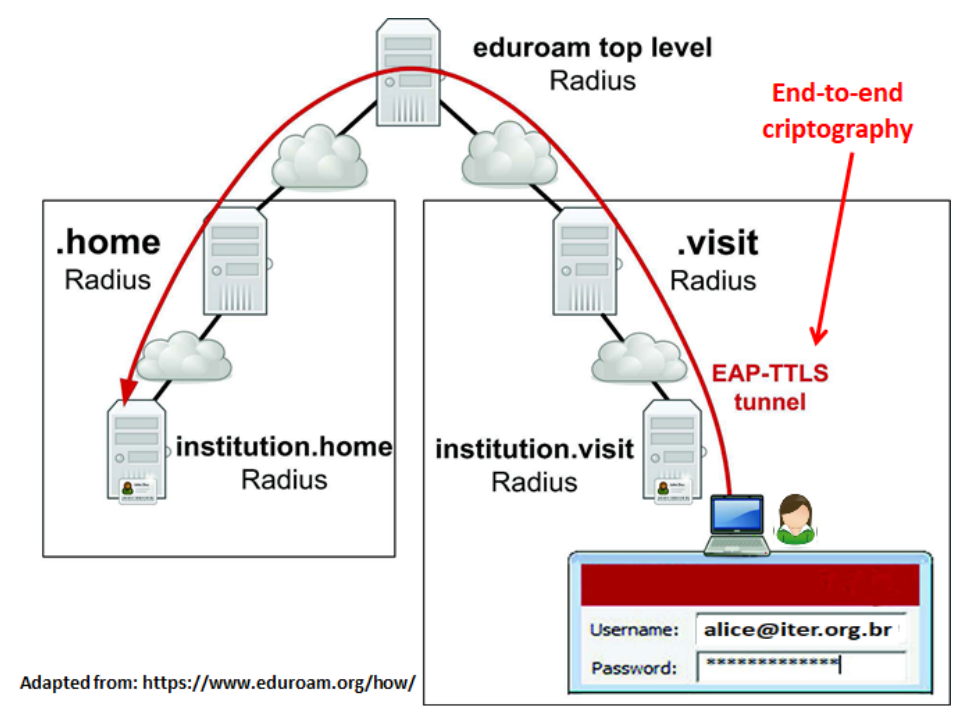

Figure 3: How eduroam works. Adapted from: https://www . eduroam.org/how 
Alice is a student with an ITER ID. She has a name associated with the project domain and a password, which we represent as a pair: [alice@iter.org.br, password]. She was visiting the library of a University in the countryside of Pernambuco and realized that there were eduroam there. This University is the institution.visit. Alice picked up the signal on her cell phone and connected to the institution.visit's RADIUS, as shown in the above figure. RADIUS did not recognize alice@iter.org.br and forwarded an encrypted message to a RADIUS from the Brazilian eduroam federation, asking if it knew alice@iter.org.br. The federation's RADIUS recognized that the institution of the iter.org.br domain was ITER and sent the request data to ITER's RADIUS, which is Alice's institution.home. This RADIUS reports directly and properly encrypted to the RADIUS of institution.visit, the information that Alice is known and that, in addition, her password was correct. Therefore, Alice surfed the Internet! This process took a few seconds...

In Brazil eduroam is managed by the National Education and Research Network ${ }^{2}$ (RNP) with more than 2,600 access points available.

\section{ITER model}

eduroam takes advantage of the techniques available on RADIUS [4] or, more effectively, available on freeRadius ${ }^{3}$ [5]. ITER takes ownership of the facilities provided by eduroam and, also, by the techniques provided by freeRadius. ITER's explanation follows the display of its model, in Figure 4.

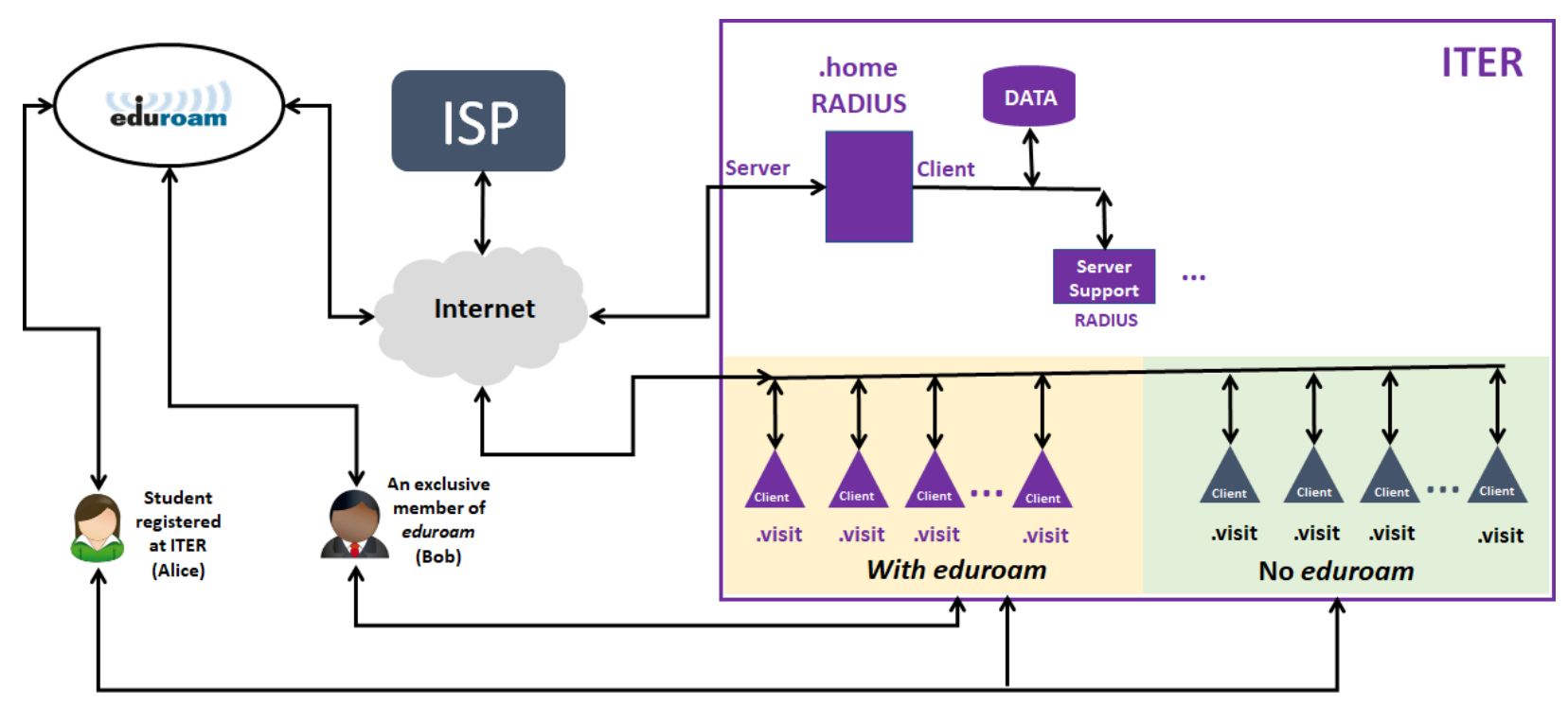

Figure 4: The ITER Project model. Source: [1].

In the purple rectangle on the right are the resources of ITER and the relationship with its users and facilities (such as eduroam), on the left. In addition to Alice, a student registered at ITER, another user appears to understand how ITER works: it is Bob, a character registered only at eduroam.

Alice, being registered with ITER, is able to use the Internet both in ITER environments associated with eduroam and in those not on eduroam but available through ISPs partners of ITER. Bob can only access the Internet via an eduroam .visit, several of which are available through ITER. This difference eases Alice's access to Bob since ITER agreed with the providers to use their immense capillarity of Internet signal diffusion. eduroam members understand each other because they have RADIUS servers, which allow for strong collaboration and interconnectivity among them. ITER also has RADIUS servers in its infrastructure, which interconnect with the RADIUS servers of the eduroam federation, in Brazil, available through RNP.

These same ITER RADIUS servers are connected to the partner ISPs' RADIUS servers, which provide access to students, such as Alice, through their numerous capillary access points with the IEEE 802.1X protocol.

\footnotetext{
${ }^{2}$ https://www.rnp.br/servicos/alunos-e-professores/colaboracao-a-distancia/eduroam

${ }^{3}$ https://freeradius.org/
} 


\section{ITER Architecture}

The ITER ecosystem is very similar to eduroam [2], with some special operational features, which can be seen in Figure 5. Actually, ITER architecture uses the best in the structure of eduroam and the facilities available through RADIUS.

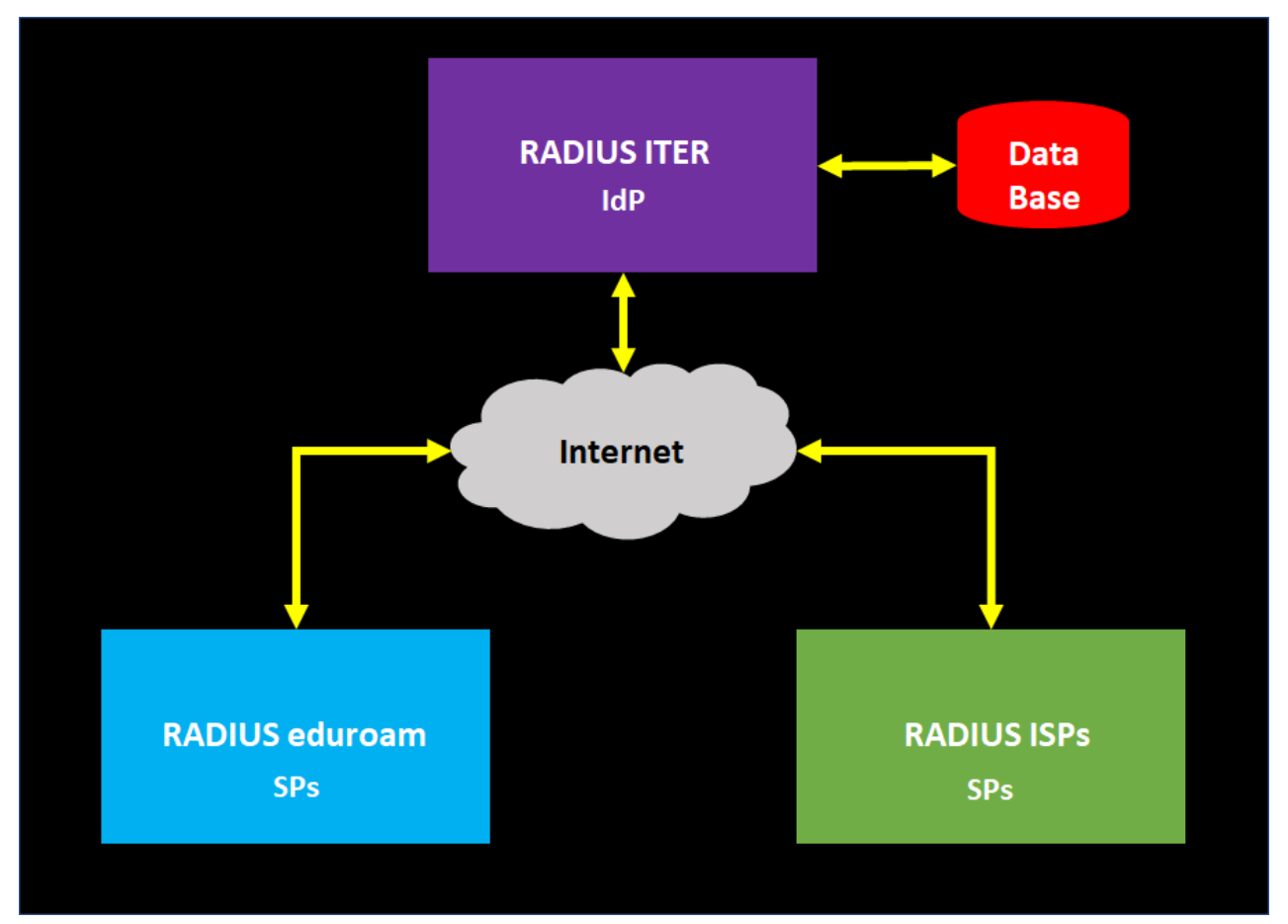

Figure 5: ITER Architecture: acts only as an Identity Provider (IdP). Source: [1].

- It serves two environments: the eduroam environment and the specific environment of ITER itself. The RADIUS servers in both environments are logically presented as a hierarchy similar to the name resolution scheme by the DNS server, wherein each environment there is a root RADIUS. In the case of eduroam each country has a root RADIUS.

- Your RADIUS at the top of the hierarchy (remote server) acts only as an Identity Provider (IdP).

- All other RADIUS, both in the eduroam environment and in the ISPs environment act as Service Providers (SPs), recognizing a student previously registered with ITER.

- The eduroam environment recognizes ITER's RADIUS through its RADIUS server at the Brazilian federation level, under the administration of RNP.

- The RADIUS servers in the ITER environment are managed by the ISPs participating in the ITER Project. They are SPs and act as proxies, in the concept of RADIUS [3]: an ISP's RADIUS server receives an authentication request from a RADIUS (for example, a Network Access Server (NAS)) and forwards directly to RADIUS ITER connecting through its domain cite RFC7593.

- The authentication mechanism is a combination of IEEE 802.1X [6]., and the Extensible Authentication Protocol (EAP) $[7,8,9,10]$. This is the authentication process for eduroam and also adopted by the ITER environment.

- This authentication mechanism carried out by a RADIUS server acting as a proxy is called supplicant and acts equally in any environment using the trust structure of eduroam using the Network Access Identifier (NAI) format (for example, alice@iter.org.br) [11, 12], used by RADIUS. The figure 6 illustrates this behavior, associating it with the EAP and the TLS protocol, inherent to RADIUS cite RFC5246, RFC6614, RFC4279 with the facilities of the Tunnel Extensible Authentication Protocol (TEAP). This tunneling method that allows secure communication between a client and a server, using TLS to establish a mutually authenticated tunnel [13]. 


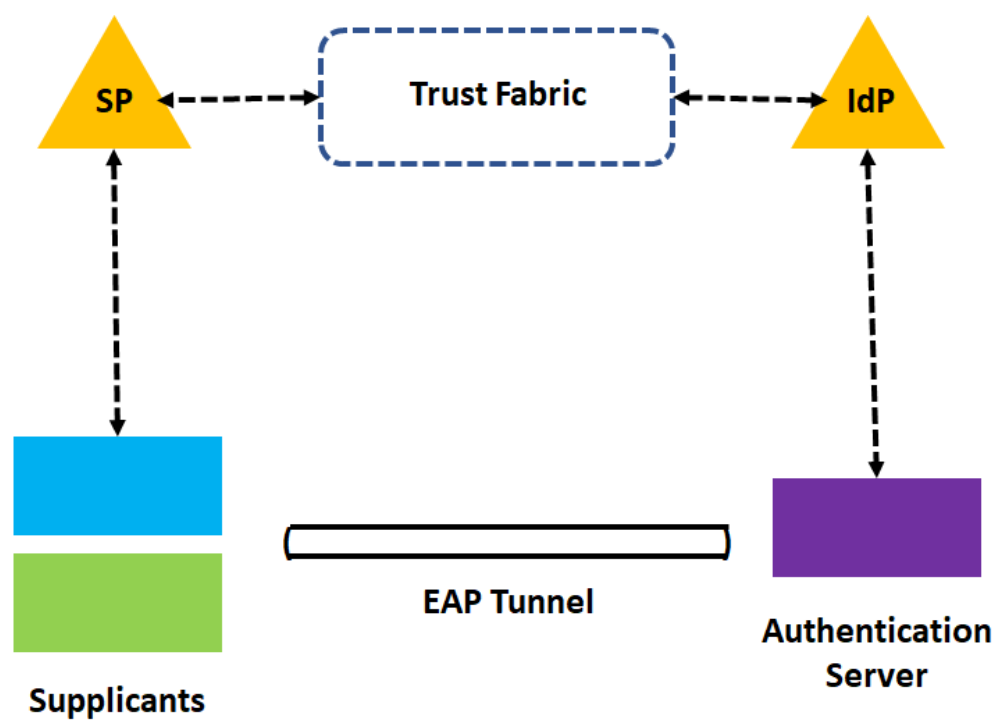

Figure 6: EAP tunneling. Adapted from RFC7593 [2]

- All attention will be paid to the security issue. Thus, the host of the ITER structure will have to ensure that it follows the recommendations and participates in the Mutually Agreed Norms for Routing Security ${ }^{4}$ (MANRS) .

\section{Operational strategies of the project}

To make ITER feasible, a set of strategies is being proposed that culminate in the most important one, named as Proof of Concept (PC).

The proposed operational strategies are listed below and described below.

- Governance

- Remuneration of ISPs

- Partial use of eduroam

- Proof of Concept

\subsection{Governance}

The ITER project is institutionally linked to ISOC Brazil, which plays advisory and supervisory roles, as well as supporting institutional relations with other entities and seeking sponsorship. But the governance of the project is done by its team, which relates to the multiple stakeholders, generically referred to as entities, intending to make it operational. The organization of the governance structure of ITER is in preparation and the Figure 7 shows the functional relationship between ISOC Brazil, the project Steeering Committee and the multiple stakeholders.

Stakeholders are volunteers, collaborators, partners and sponsoring organizations. All of them come together to ensure that ITER's objective is realized: serving the students of elementary and high school courses, the main interested party, and called target. Grouped in an organized manner by the project's coordination committee, under the institutional shelter of ISOC Brazil, the entities interfere in the organization of ITER, through a process that allows recommendations to change the statutes, similar to the participation of society in Internet policies for Latin America and the Caribbean promoted by the Internet Address Registry Latin America and the Caribbean ${ }^{5}$ (LACNIC).

The volunteers are the entities (people, institutions, organizations, etc.) that provide partial or total work to the project, without remuneration. They do this permanently or temporarily. The acceptance and evaluation of volunteers for ITER is the responsibility of the project's Steering Committee.

${ }^{4}$ https : //www . manrs .org/

${ }^{5}$ https://www.lacnic.net/1042/3/lacnic/acerca-do-lacnic 


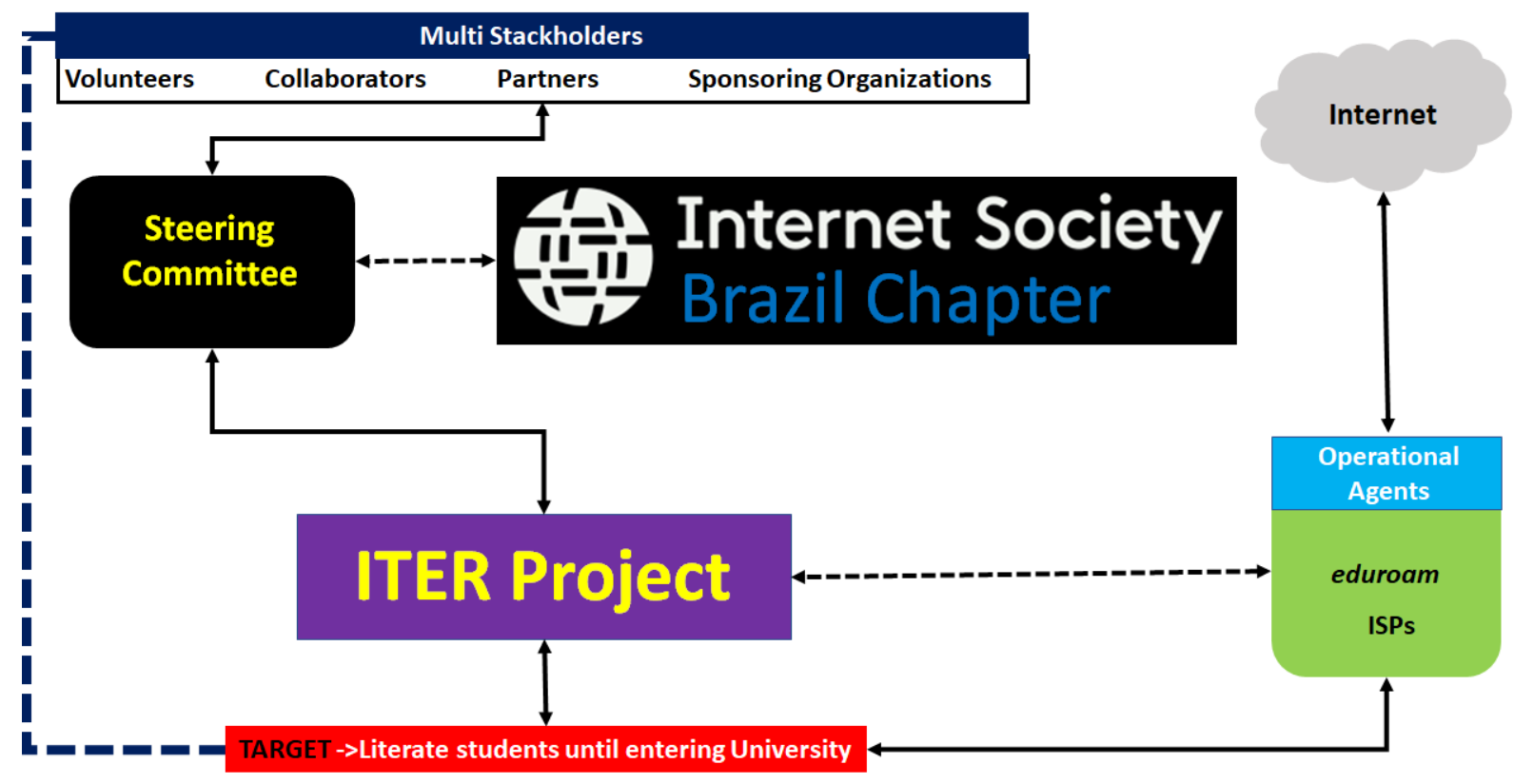

Figure 7: The governance of the ITER Project. with its Steering Committee, the institutional support of ISOC Brazil and the multiple stakeholders. Source: [1].

The collaborators are the entities (people, institutions, organizations, etc.) who exercise their skills in ITER demands and are paid for their work. The administration of the collaborators is responsibility of the Steering Committee.

The partners are the main stakeholders in the project from an operational point of view. They include schools (public and private) from elementary education to vocational education and also ISPs. The Steering Committee is responsible for calling, registering and providing guidance and, when appropriate, remunerating partners.

The sponsoring organizations are responsible for financing ITER, that is, the partners' remuneration, investments and operating costs projected by the Steering Committee.

Entities can participate in one or more interested parties and relate to each other as in Figure 8 becoming uncharacterized, at the intersection of all of them.

\subsection{ISPs remuneration}

In March 2020, the Brazilian National Communications Agency (Anatel) announced that small ISPs are placing themselves and a comfortable position among the major operators in the sector. This evolution over the years can be seen in Figure 9.

With this, they are, over time, increasing the capillarity, making available access to the Internet, and the most remote Brazilian territory.

For ITER they are the ideal partners and complementary to those of the big providers.

As a way of expanding capillarity more quickly and reaching strategic locations from the point of view of serving the target they need and should be adequately remunerated. The Coordination Committee, where a representative of the ISPs will be present, will establish the appropriate and fair remuneration to the ISPs after running the PC.

\subsection{Partial use of eduroam}

eduroam is an opportunity to make immediate access to the internet available to target through its more than 2,600 points spread across Brazil. It depends only on understanding with RNP.

\subsection{Proof of Concept}

Some volunteers are developing protocols to establish a test bed, which is called Proof of Concept (PC). 


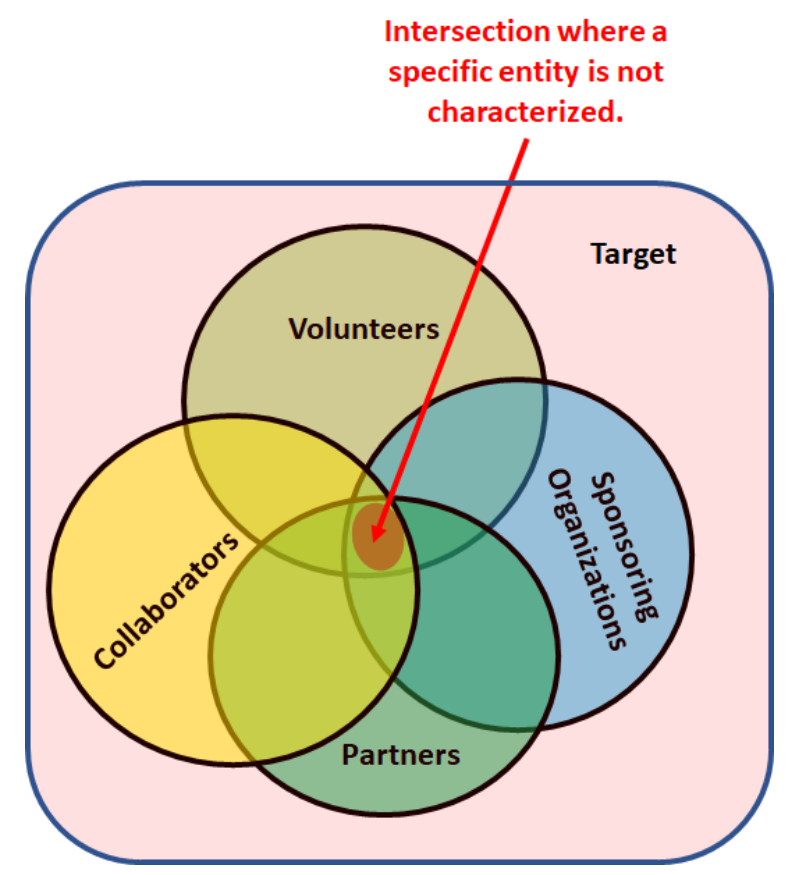

Figure 8: An entity can participate characterized as several stakeholders. So at the intersection it is not characterized. Source: [1].

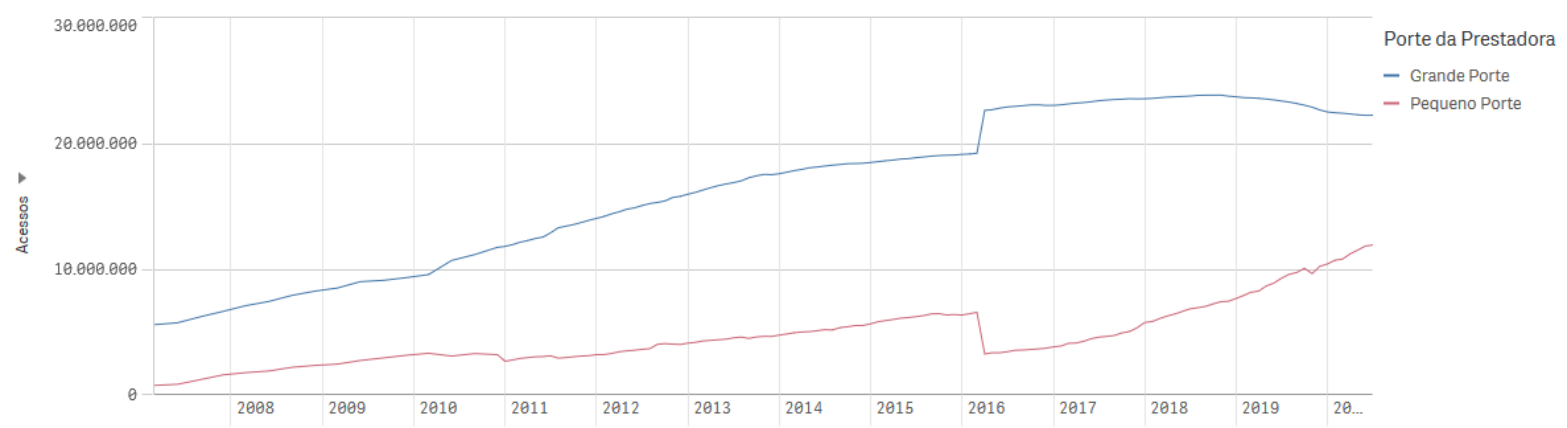

Figure 9: Evolution of fixed broadband accesses by provider and company size. The 10,405 entities registered with Anatel, produced a total of 34,181,324 accesses, large providers account for 22,249,889 accesses $(65.10 \%)$ and small providers account for 11,931,455 accesses (34.90\% ) in July 2020, Source: https : //www . anatel .gov.br/paineis/ acessos/banda-larga-fixa

The PC for ITER is the operation for a period of one year, of the integral model proposed in Figure 4. The steps involved in preparing to run $\mathbf{P C}$ are as follows:

1. Convocation of interested ISPs who have RADIUS in their infrastructure and are willing to participate in this first year of the $\mathbf{P C}$, without remuneration;

2. Calling primary and secondary schools, particularly from remote locations but with Internet coverage through an ISP;

3. Convocation of a volunteer to participate in the debates that will establish the technical definitions;

4. Non-governmental institutions that can guide the team of volunteers to establish policies to periphery approach;

5. Convocation of manufacturers and / or representatives of equipment manufacturers adhering to the ITER project, such as the Network Access Server (NAS);

6. Sponsoring Organizations interested in participating in the rules that will outline ITER's future and how financial contributions would be made to maintain traffic via ISPs, after PC. 


\section{Schedule of initial activities}

Table I identifies the main components needed to leverage ITER and its PC. Like any project, these components change over time, therefore requiring permanent project coordination.

Table 1: Preliminary schedule of the main steps involving ITER.

\begin{tabular}{|r|l|l|l|l|}
\hline$\#$ & \multicolumn{1}{|c|}{ Activity } & \multicolumn{1}{c|}{ Responsibility } & \multicolumn{1}{c|}{ Start } & \multicolumn{1}{c|}{ End } \\
\hline 1 & Technical project of PC & $\mathrm{JB}^{a}, \mathrm{LL}^{b}, \mathrm{MS}^{c}, \mathrm{JN}^{d}$ & $\mathrm{Sep} / 20$ & $\mathrm{Oct} / 20$ \\
\hline 2 & Operation of PC & $\mathrm{JB}^{a}, \mathrm{LL}^{b}, \mathrm{MS}^{c}, \mathrm{JN}^{d}$ & $\mathrm{Nov} / 20$ & $\mathrm{Oct} / 21$ \\
\hline 3 & Project ITER operation & Steering Committee & $\mathrm{Oct} / 21$ & - \\
\hline 4 & Marketing Plan & Steering Committee & $\mathrm{Sep} / 20$ & - \\
\hline 5 & Unique Identification (Warranty) & Steering Committee & $\mathrm{Sep} / 20$ & $\mathrm{Oct} / 20$ \\
\hline 6 & ISPs partnership agreement for PC period & Steering Committee & $\mathrm{Sep} / 20$ & $\mathrm{Sep} / 20$ \\
\hline 7 & Call Participation for ISP Partners & Steering Committee & $\mathrm{Oct} / 20$ & - \\
\hline 8 & Call for Volunteer Participation & Steering Committee & $\mathrm{Sep} / 20$ & - \\
\hline 9 & First ITER policy document & Steering Committee & $\mathrm{Sep} / 20$ & $\mathrm{Oct} / 20$ \\
\hline 10 & Policy document change rules & Volunteer Group & $\mathrm{Sep} / 20$ & $\mathrm{Oct} / 20$ \\
\hline 11 & ITER's Governance Structure & Steering Committee & $\mathrm{Sep} / 20$ & - \\
\hline 12 & 19/5000 ITER website & Steering Committee & $\mathrm{Sep} / 20$ & - \\
\hline 13 & Involvement of Sponsoring Organization's actions & Steering Committee & $\mathrm{Sep} / 20$ & - \\
\hline
\end{tabular}

Legend:

$a_{\rightarrow}>$ Julião Braga

${ }^{b} \rightarrow>$ Luciano I. Lima

$c^{c}>$ Marcelo Santos

$d_{->\text {Jeferson Nobre }}$

\section{Conclusions and Future Work}

Sponsoring Organizations will only venture to support ITER, if PC demonstrates the effectiveness of the results. This indicates the need for special attention during the testing stage, which should have mechanisms to assess direction changes in the face of possible difficulties in current actions.

It is to be expected that one of the major beneficiaries of the reduction of school inequality, in the medium and long term, will be higher education institutions, especially private universities. So they are strong candidates for interested parties like Sponsoring Organizations.

One of the concerns that will be part of the PC stage is the efficiency of the Radius server (s) available for authentication, since the database will use SQL, a reasonably slow mechanism, mainly in data writing. Additionally, other factors affect the efficiency of Radius servers, which include: availability of main memory, CPU speed, the EAP-TTLS mechanism and the complexity of the implemented policies ${ }^{6}$. A team will be available with the objective of simulating and measuring the efficiency of configurations that should be introduced in the ITER environment in its normal operational activity, which will be able to serve 39 million students - estimated by Brazilian Ministry of Education [14] —, under the AAAA protocol ${ }^{7}$. Radius (and freeRadius) has efficiency simulation features in various scenarios that can be designed with ITER, in the radclient tool and in others developed by third parties, such as $\operatorname{RadPerf}^{8}$. On the other hand, a Radius server can behave as a client to another Radius server, which ensures modular scalability.

Other types of partners will exist and techniques for participation by these new partners will be needed. For example, public and private schools with a navigable Internet band can participate in eduroam, as long as this is permitted by their governance in Brazil or directly at ITER. Such partners use the Internet as a means and, therefore, will not require operational costs from ITER, but technical support.

\section{Acknowledgments}

The authors would like to thank:

\footnotetext{
${ }^{6}$ https://wiki.freeradius.org/features/ Fast

${ }^{7}$ Implements authentication, authorization, accounting and auditing services

${ }^{8}$ https://networkradius.com/radius-performance-testing/
} 
- Professor Dr. Flavio Rech Wagner (ISOC Brasil, UFRGS), for the suggestions that clarify the support of ISOC Brazil o the Iter Project;

- Professor Dr. Demi Getschko (ISOC Brasil, PUC-SP), for supporting the project, recommendations, and guidelines based on his incomparable experience;

- Engineer Luciano Inacio Gonçalves Lima, Technical Director of Jupiter Telecomunicacoes for the availability of material resources necessary for the execution of PC.

\section{References}

[1] Juliao Braga1, Ana Leda Silva Moraes, Jéferson Nobre, and Marcelo Santos. Diminuindo a desigualdade no acesso à Internet entreestudantes alfabetizados até a entrada na Universidade. In Anais do VI Workshop Pré-IETF To be published. SBC, 2020.

[2] K. Wierenga, S. Winter, and T. Wolniewicz. The eduroam Architecture for Network Roaming. Technical report, RFC Editor, September 2015. DOI: 10.17487/RFC7593.

[3] C. Rigney, S. Willens, A. Rubens, and W. Simpson. Remote Authentication Dial In User Service (RADIUS). Technical report, RFC Editor, June 2000. DOI: 10.17487/RFC2865.

[4] Jonathan Hassell. RADIUS: securing public access to private resources. " O’Reilly Media, Inc.", 2002.

[5] Dirk Van der Walt. FreeRADIUS Beginner's Guide. Packt Publishing Ltd, 2011.

[6] IEEE. IEEE Standard for Local and metropolitan area networks - Port-Based Network Access Control. Technical report, IEEE, 2010. DOI 10.1109/ieeestd.2010.5409813, http://ieeexplore.ieee.org/servlet/opac?punumber=5409757.

[7] F. Adrangi, A. Lior, J. Korhonen, and J. Loughney. Chargeable User Identity. Technical report, RFC Editor, January 2006. DOI: 10.17487/RFC4372.

[8] B. Aboba, L. Blunk, J. Vollbrecht, J. Carlson, and H. Levkowetz. Extensible Authentication Protocol (EAP). Technical report, RFC Editor, June 2004. DOI: 10.17487/RFC3932.

[9] H. Alvestrand. The IESG and RFC Editor Documents: Procedures. Technical report, RFC Editor, October 2004. DOI: $10.17487 /$ RFC3748.

[10] D. Stanley, J. Walker, and B. Aboba. Extensible Authentication Protocol (EAP) Method Requirements for Wireless LANs. Technical report, RFC Editor, March 2005. DOI: 10.17487/RFC4017.

[11] S. Winter and M. McCauley. Dynamic Peer Discovery for RADIUS/TLS and RADIUS/DTLS Based on the Network Access Identifier (NAI). Technical report, RFC Editor, October 2015. DOI: 10.17487/RFC7585.

[12] A. DeKok. The Network Access Identifier. Technical report, RFC Editor, May 2015. DOI: 10.17487/RFC7542.

[13] H. Zhou, N. Cam-Winget, J. Salowey, and S. Hanna. Tunnel Extensible Authentication Protocol (TEAP) Version 1. Technical report, RFC Editor, May 2014. DOI: 10.17487/RFC7170.

[14] INEP-MEC. Censo Escolar. http://portal.inep.gov.br/censo-escolar. Accessed: 2020-09-09. 